\title{
DESIGUALDADES EN LA OFERTA DE EMPLEO SEGÚN REGIONESY CATEGORÍAS URBANAS. ARGENTINA (2010)
}

\section{Fernando Ariel Manzano}

\section{Guillermo Angel Velázquez}

Universidad Nacional de Córdoba - UNC - Córdoba - Argentina

\begin{abstract}
Resumo: En este trabajo exploramos la desigualdad de la oferta laboral y la determinación que sobre ella ejercen la escala urbana y el contexto regional en Argentina. Buscamos verificar la siguiente hipótesis: A mayor jerarquía urbana (teniendo en cuenta las diferencias regionales en términos de estructura productiva y ocupacional), se elevan la tasas de actividad total, que generan el incremento de la participación económica femenina, además se evidencia el mejoramiento de otros indicadores -reducción de la relación de dependencia demográfica infantil y aumento del nivel educativo de la fuerza laboral-. Las variables del mercado de trabajo utilizadas están sujetas a la disponibilidad de información sobre la temática del último censo nacional argentino del año 2010, que representa la base empírica de esta investigación.
\end{abstract}

Palavras-chave: Oferta de empleo. Sexo. Jerarquía Urbana y Regiones.

\begin{abstract}
In this work we explore the inequality of the labor supply and the determination that on her exercise the urban scale and the regional context in Argentina. We seek to verify the following hypothesis: to greater urban hierarchy (taking into account regional differences in terms of productive structure and occupational), raise the rates of total activity, which generate the increase in the economic participation of women, in addition to evidence the improvement of other indicators -the reduction of the dependency ratio aumentodel child demographic and educational level of the labor force-. Labor market variables used are subject to the availability of information on the theme of the last national census Argentina 2010, that represents the empirical basis for this research.
\end{abstract}

Keywords: Job offer. Sex. Urban hierarchy and regions. 


\section{Introducción}

La evolución de la dinámica demográfica de la Argentina muestra profundos cambios a lo largo de la historia, tanto en el nivel y ritmo del crecimiento como en el papel que jugaron los factores que la determinan.

En términos de redistribución espacial, Argentina presenta un proceso de urbanización temprano en relación a los países de la región, la proporción de población que vive en localidades urbanas ha protagonizado un incremento constante a lo largo del tiempo, con un crecimiento muy marcado hasta 1970.

Según Recchini y Lattes (1975) a principios del siglo pasado la localización preferencial de los migrantes europeos ocurrió en "las áreas de Buenos Aires en primer término y luego las del Centro-Litoral fueron aquellas en las que el impacto de la migración internacional fue mayor" (p. 58), dejando como resultado una alta concentración poblacional en el Área Metropolitana de Buenos Aires (en adelante AMBA), agravada posteriormente con el aporte masivo de los flujos migratorios internos.

$\mathrm{Si}$ bien suele coincidirse en que aproximadamente a partir de 1960, el crecimiento poblacional total depende casi exclusivamente del crecimiento vegetativo, debemos tener en cuenta que los niveles de fecundidad y de mortalidad presentan profundas diferencias entre las regiones o áreas de residencia del país. Las corrientes migratorias internas de predominancia femenina hacia las grandes ciudades -"asociadasa una mayor existencia de oportunidades económicas en relación con el trabajo femenino en las poblaciones urbanas que en las rurales" (RECCHINI y LATTES,1975, p. 139-140), compensan parcialmente las menores tasas de fecundidad de las grandes ciudades receptoras ,así como también tiende a retardar su nivel de envejecimiento.En las restantes regiones, conforme transcurre el tiempo, también avanza la tendencia hacia el incremento de los niveles de urbanización esto hace descender el porcentaje de participación del AMBA en el total urbano del país, lo cual no implica una disminución de la población de este aglomerado en términos absolutos-. El proceso de urbanización de Argentina se destaca por la heterogeneidad de sus resultados a nivel provincial y regional -combinando diferentes niveles de primacía de la ciudad más importante en relación con las restantes vinculadas-, mediante desigualdades en el ritmo del crecimiento y en el porcentaje de población urbana. Por tanto, se presentan situaciones extremas de alta concentración urbana, así como casos provinciales de niveles de urbanización más equitativos, en donde se destaca el crecimiento de las ciudades intermedias. En síntesis, el estado de situación expresa la creciente complejidad de la redistribución espacial de la población en el territorio argentino.

Consideramos que las desigualdades en la distribución de la oferta de empleo -en términos cuantitativos como cualitativos-, se encuentran vinculadas e interrelacionadas con este proceso de urbanización desigual a escala provincial y regional.

Los indicadores de oferta de empleo a nivel del promedio nacional no son representativos, y ocultan las diferencias importantes a nivel regional -como también provincial-. Sobresalen marcadas diferencias a nivel regional respecto a la tasa de participación de la fuerza de trabajo, destacándose históricamente las regiones Pampeana y Patagónica por una mayor contribución de la población en la actividad económica.

Además de esta vinculación espacial a nivel agregado entre el factor regional y la tasa de participación de la fuerza de trabajo, consideramos como relevante la influencia de la escala urbana.

La hipótesis que buscamos corroborar es que el incremento de la tasa de empleo total tiene una relación directa con el grado de jerarquía urbana, mediante la incidencia en el aumento de la participación laboral femenina en actividades de servicios -complementarias de las actividades primarias y secundarias, predominantes a escalas geográficas de menor densidad económica-. Del lado de la demanda laboral, conforme se incrementa el nivel de jerarquía urbana en cada región, se evidencia 
una mayor diversificación de sectores productivos en cada región. Mientras que por el lado de la oferta laboral, se incrementa la sensibilidad a participar del mercado laboral, así como una mayor segmentación y especialización en los perfiles ocupacionales-se produce una adaptación a nuevos tipos de ocupación, que exigen una mayor inversión en capital humano-.

A medida que aumenta el nivel de escala urbana de las diferentes regiones, tiende a generarse un incremento de la diversificación de las estructuras productivas, dando lugar a un aumento del peso relativo de las actividades terciarias, por sobre las actividades primarias y secundaria, sesgando la demanda de empleo hacia una mayor participación laboral femenina -no siempre sustitutivas de las actividades laborales masculinas-, y por ende generando como resultado un incremento de la oferta total de empleo.

En el apartado que sigue a continuación, se realizarán algunas aclaraciones metodológicas respecto a la información ocupacional de la personas que releva el operativo del censo de población, describiremos el uso de los conceptos operacionales que se construyen a partir de sus datos - y las limitaciones de los mismos-, y también presentaremos la evolución de los indicadores clásicos del mercado laboral a nivel de total país entre los años 1869 y 2010. En el apartado N³, nos dedicamos a analizar el comportamiento de algunos indicadores demográficos vinculados a la partición laboral (relación de dependencia demográfica infantil, proporción entre cantidad de trabajadores potencialmente activos respecto de inactivos), en función del tamaño de los aglomerados urbanos a nivel regional y total país. En el apartado $\mathrm{N}^{\circ} 4$, profundizamos en el comportamiento de la oferta de empleo según sexo, y la influencia que genera la segmentación del espacio territorial en función del nivel de jerarquía urbana. En el ante último apartado, nos abocamos a la relación entre el nivel educativo máximo alcanzado por la población activa, considerando los cambios según la diferenciación entre distintos niveles de jerarquía urbana en cada una de las regiones. En el último apartado, se expresan de manera sintética los principales resultados que contiene esta investigación.

\section{Consideraciones metodológicas y comportamiento historico de la oferta $y$ demanda de empleo en Argentina.}

Conforme la matriz legalista de los $\operatorname{censos}^{1}$, la información del bloque ocupación es relevada sobre las personas de 14 años y más, por definir esa edad el fin del periodo escolar obligatorio para el Estado ${ }^{2}$ (OTERO, 1999). La pregunta sobre condición de actividad $^{3}$ nos permite dividir en dos grupos a todas aquellas personas de 14 años y más. Un primer grupo formado por las personas que se encuentren trabajando o deseen trabajar en un momento determinado, denominados como "población económicamente activa" -en adelante PEA- y un segundo grupo formado por la población restante mayor de 14 años, que corresponde a las personas no económicamente activas -en adelante PNEA-.

La PEA a nivel total del país representa la oferta de empleo visible de hombres y mujeres dispuestos a trabajar en el sistema económico. Esta depende, por un lado, del crecimiento vegetativo de la población y de los cambios migratorios con otros países, y por otro, de los cambios en las condiciones de la demanda de trabajo que modifican la situación de "equilibrio de la oferta laboral". Además debemos adicionar a todas las personas que trabajan por su cuenta y aquellas otras personas que tienen gente a

\footnotetext{
1 Por legalismo se entiende la tendencia de los sistemas estadísticos a estudiar la realidad social a partir de definiciones jurídicas codificadas por el Estado y no mediante definiciones sociológicas más sensibles a la prácticas sociales efectivas (OTERO, 1999).

${ }^{2}$ La inadecuación del precepto legalista es particularmente evidente en este punto, ya que la edad al inicio de la actividad laboral era en muchos casos inferior a la establecida por la ley. Una situación típica a este respecto, entre muchas otras referencias posibles, es ilustrada por CACOPARDO y MORENO (1997).

${ }^{3}$ Cabe destacar que la condición de actividad, que clasifica a la población en económicamente activa (PEA) e inactiva (PNEA), permite una aproximación al mercado de trabajo por medio de la tasa de actividad y al desempleo, a través de la tasa de desocupación. En ese sentido, la captación de la condición de actividad no es menor, ya que el método de recolección de la información se ve influida por la evaluación de los encuestadores y la percepción subjetiva de los entrevistados, y ambas a su vez se encuentran condicionadas por construcciones sociales, decisiones teóricas en la elaboración del cuestionario y estereotipos socioculturales.
} 
su cargo. Este agregado representa el total de las fuerzas de trabajo que existen en un país, en un momento determinado.

Dentro de la PEA se distinguen habitualmente dos subconjuntos: el de los ocupados -quienes desempeñan alguna actividad económica- y los desocupados -definidos como aquellos que buscan activamente un empleo-. Así presentados, estos dos subconjuntos resultan excesivamente genéricos. Para llevar adelante un análisis más detallado y poder comprender mejor la dinámica del mercado de trabajo, se requiere de mayor especificidad y desagregación de la información. En general, el número total de ocupados ofrece una sobrerepresentación de ese grupo, puesto que no distingue entre quienes trabajan una cantidad de horas que pueden considerarse normal -entre 35 y 44 horas semanales según la pauta social prevaleciente- y quienes sólo trabajan a tiempo parcial pero desearían trabajar más; a estos últimos se los conoce como sub-ocupados o parcialmente desocupados y sólo pueden considerarse como ocupados, debido a que la información censal no permite diferenciarlos. Sería importante conocer hasta qué punto una proporción relativamente importante de la fuerza de trabajo opta por empleos a tiempo parcial de manera voluntaria, y cuántos, de existir la posibilidad, aumentarían su jornada laboral o su intensidad -según sea la paga en el tipo de trabajo-, o bien estarían interesados en sumar otro empleo.

Con respecto al número total de desocupados, se suele considerar, en cambio, que existe una subrepresentación de este conjunto, debido a que sólo distingue a aquellos que buscan trabajo, sin relevar a quienes se retiran del mercado laboral o no buscan trabajo, dadas las malas condiciones laborales existentes. Estos últimos constituyen la desocupación "invisible" o no manifiesta y representarían una oferta potencial de trabajadores disponibles si perciben mejoras en las condiciones del mercado de trabajo actual. Este grupo de trabajadores, metodológicamente, presenta mucha dificultad para lograr cuantificarlo (PALOMINO, 1988).
El otro componente del mercado de trabajo corresponde a la demanda de empleo, que representa el agregado de la cantidad de trabajadores que las empresas o empleadores están dispuestos a contratar. La demanda de empleo depende también de las potencialidades de la oferta de empleo, de modo tal que esta última pueda satisfacer los requerimientos necesarios en cuanto a nivel educativo, conocimiento tecnológico, antigüedad de tiempo en el oficio; así como otras características necesarias para ser incorporado al proceso productivo. Es decir, la oferta y demanda de trabajo, tienen en común muchos elementos que determinan su comportamiento de manera interdependiente.

En síntesis, el mercado de trabajo se ve afectado por los cambios en la participación de la población de 14 años y más sobre el total -variable netamente demográfica-; la variación de la PEA -la decisión de toda persona que haya alcanzado los 14 años de edad a participar del mercado laboral- y las variaciones de la cantidad de población ocupada -resultado de la interacción de la oferta y demanda de empleo-.

Es posible realizar una cuantificación de estos factores mediante tres indicadores del mercado laboral, que presentan históricamente marcadas diferencias en cuanto a su variabilidad para el caso del mercado laboral argentino.

En la Figura 1 se observa que entre el año 1869 y 2010 para el total de Argentina, la tasa de crecimiento medio anual -en adelante TCMA- de la "población mayor de 14 años" presenta el comportamiento más estable -utilizando como medida de dispersión el coeficiente de variación ${ }^{4}$ (C.V.)-, su valor es de sólo $11,0 \%$ durante este periodo de 141 años. Su baja dispersión se debe a que se encuentra afectada solamente por factores demográficos -natalidad, mortalidad y migracionesque suelen presentar modificaciones más tenues en relación a las restantes variables socio-económicas vinculadas al mercado de trabajo. La TCMA de la PEA durante el periodo 1869-2010, presenta un C.V.

\footnotetext{
4 El coeficiente de variación, permite comparar el nivel de variabilidad existente entre grupos de datos referidos a distintos sistemas de unidades.
} 
de $34,5 \%$-casi tres veces superior a la variabilidad que presenta el crecimiento de la "población de 14 años y más"-. Mientras que la población ocupada presenta un comportamiento aún mucho más errático, su C.V. alcanza el 117,0\% -superando en más de 10 veces la variabilidad de la TCMA de la población de 14 años y más y en más de 3 veces a la PEA-, debido a que esta depende básicamente de los cambios en las condiciones de la actividad económica que llevan a contratar más o menos mano de obra y a su interacción con la oferta de empleo.

Figura 1- Tasa media anual de Población mayor a 14 años, Población Ocupada y PEA (en $\%)$. Periodos seleccionados: 1869-1895; 1895$1914 ; 1914-1947 ; 1947-1960 ; 1960-1970 ; 1970-$ 1980; 1980-1991; 1991-2001 y 2001-2010.

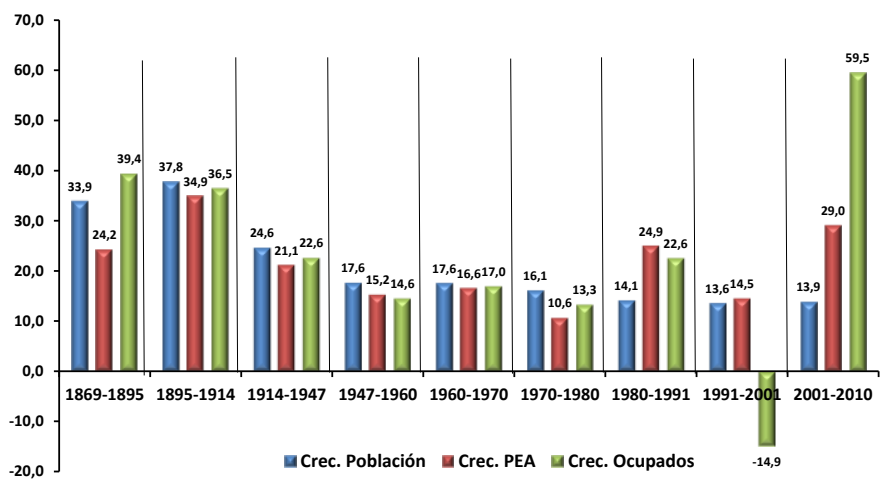

Fuente: Elaboración personal en base a datos de los censos de población (INDEC, 1974, 1981, 1992, 2004, 2012; dirección de estadística e investigaciones económicas de mendoza, 2016).
Como comentamos anteriormente, el objetivo de esta investigación es analizar el efecto de la introducción de la división espacial según distintas jerarquías de escala urbana sobre la oferta de empleo.

Para operacionalizar la segmentación en jerarquías urbanas del territorio argentino, haremos uso de la tipificación presentada en el clásico trabajo de Vapñarsky y Gorojovsky ${ }^{5}$ (1990). Mientras que para realizar la división regional de la Argentina, seguiremos la propuesta del Instituto Nacional de Estadística y Censos a partir del VII censo nacional (1980) basada en límites políticos provinciales ${ }^{6}$-salvo para el caso de la Región Metropolitana-, (VELÁZQUEZ, 2008).

En laTabla1podemos observar el resultado de la implementación del entrecruzamiento de la segmentación según niveles de jerarquía urbana y la división regional. La característica saliente que presenta el territorio argentino, es la alta desigualdad respecto a la distribución poblacional.
Tabla 1-Distribución porcentual de la población total en categorías urbanas y regiones. Argentina (2010).

\begin{tabular}{|c|c|c|c|c|c|c|c|c|}
\hline \multirow{2}{*}{ Jerarquía } & \multirow{2}{*}{ Tamaño (hab) } & \multicolumn{7}{|c|}{ Cantidad de Poblacion (en \%) } \\
\hline & & NEA & NOA & Cuyo & Metropolitana & Pampeana & Patagonia & Total \\
\hline Metrópolis y Ciudades Grandes & $>1.000 .000$ & & & & 33,9 & 6,6 & & 40,5 \\
\hline ATIS grandes & $400.000-999.999$ & 1,8 & 3,4 & 3,4 & & 4,7 & 1,0 & 14,3 \\
\hline ATIS medias & $50.000-399.999$ & 2,2 & 3,2 & 1,2 & & 7,0 & 1,7 & 15,4 \\
\hline ATIS pequeñas & $20.000-49.999$ & 1,4 & 1,2 & 0,4 & & 4,3 & 1,0 & 8,3 \\
\hline Pueblos grandes & $2.000-19.999$ & 1,9 & 2,1 & 0,8 & & 6,5 & 1,0 & 12,4 \\
\hline Pueblos pequeños y población rural & $1-1.999$ & 1,8 & 2,3 & 1,2 & & 3,2 & 0,5 & 9,0 \\
\hline Total & & 9,2 & 12,2 & 7,1 & 33,9 & 32,3 & 5,3 & 100,0 \\
\hline
\end{tabular}

Fuente: Elaboración personal (REDATAM) sobre la base de censo 2010 (INDEC, 2012)

\footnotetext{
${ }^{5}$ Las seis categorías establecidas fueron: Metrópolis y Ciudades Grandes (más de 1.000 .000 de habitantes); ATIS (aglomeraciones de tamaño intermedias) grandes (400.000999.999 habitantes); ATIS medias (50.000-399.999 habitantes); ATIS pequeñas (20.000-49.999 habitantes); Pueblos grandes (2.000-19.999 habitantes) y Pueblos pequeños y población rural (1-1.999habitantes) (VAPÑARSKY y GOROJOVSKY, 1990).

${ }^{6}$ La propuesta incluye las siguiente división regional: Noroeste (NOA), Noreste (NEA), Cuyo, Pampeana, Metropolitana y Patagónica (VELÁZQUEZ, 2008).
} 
Las metrópolis y ciudades grandes -consideradas como aquellas que superan el millón de habitantes-, son la categoría urbana de Argentina que concentra la mayor cantidad de población del país; en ellas se asienta el 40,5\% de la población total. Este nivel de primacía urbana solo está presente en dos regiones de Argentina (Metropolitana y Pampeana).

La segunda categoría urbana de mayor peso demográfico son las aglomeraciones de tamaño intermedio (en adelante ATIS), de tamaño medias -estas comprenden un población de entre 50.000 y 399.999 habitantes-, en donde reside el 15,4\% de la población total. Se encuentran presentes en todas las regiones, con excepción del Área Metropolitana y su distribución entre las regiones es la segunda más equilibrada de las seis categorías utilizadas -su C.V. presenta un valor de $86,3 \%$ -

La tercer categoría urbana con mayor población relativa son las ATIS grandes -donde habitan entre 400 mil y 999 mil personas- y concentran el $14,3 \%$ de la población total. Se destacan por presentar la distribución regional más homogénea (C.V. de 66,4\%).

El cuarto lugar en participación pertenece a los pueblos grandes -que concentran entre 2.000 y 19.999 habitantes-, en ellos reside el $12,4 \%$ de la población nacional y poseen un alto nivel de heterogeneidad regional, su C.V. alcanza el 102,0\%-valor sólo superado por la máxima categoría de jerarquía urbana, que son las metrópolis y ciudades grandes-.

Finalmente, en la categoría correspondiente a los pueblos pequeños y población rural, que poseen menos de 2.000 habitantes, se encuentra el 9,0\% de la población total de Argentina, siendo la segunda categoría más homogénea, su C.V. es de 72,0\%

\section{El tamaño de la pea, según regiones y categorías urbanas.}

La PEA se puede relacionar con el conjunto de la población de diversas maneras. La tasa "bruta" de actividad es el cociente entre la PEA y la población total expresado en forma porcentual; es evidente que ella depende, en buena medida, de la estructura de edades de la población en cuestión, puesto que los menores de 14 años no trabajan.

El índice de dependencia demográfica de los menores de 14 años, se define como el número de menores dependientes, por cada cien personas potencialmente activas - es decir, la población de14 años y más-.

El índice de dependencia de los menores está atado a los cambios en la estructura de edad de la población y por tanto, presenta una significativa variabilidad entre las diferentes regiones y categorías urbanas.

En la Tabla 2 podemos apreciar la existencia de una relación inversa entre el valor de este indicador y las jerarquías urbanas. El valor mínimo de la tasa de dependencia demográfica de los menores se encuentra en las ciudades grandes, con un valor de 43,7 infantes por cada 100 personas potencialmente activas, mientras que el valor máximo se ubica en los pueblos pequeños, con un valor promedio de 74,8 dependientes menores por cada cien personas en edad laboral.

Tabla2: Tasa de dependencia de población infantil*, según categorías urbanas y regiones. Argentina 2010.

\begin{tabular}{|c|c|c|c|c|c|c|c|c|}
\hline \multirow[b]{2}{*}{ Categorías urbanas } & \multirow[b]{2}{*}{ Tamaño (hab) } & \multicolumn{7}{|c|}{ Tasa de dependencia de la poblacion infantil } \\
\hline & & NEA & NOA & Cuyo & $\begin{array}{c}\text { Metropolita } \\
\text { na }\end{array}$ & Pampeana & Patagonia & Total \\
\hline Metrópolis y Ciudades Grandes & $>1.000 .000$ & & & & 43,7 & 43,7 & & 43,7 \\
\hline ATIs grandes & $400.000-999.999$ & 55,0 & 56,7 & 51,3 & & 43,8 & 47,2 & $\mathbf{5 0 , 0}$ \\
\hline ATIS medias & $50.000-399.999$ & 64,4 & 58,9 & 52,8 & & 48,5 & 51,4 & 53,3 \\
\hline ATIS pequeñas & $20.000-49.999$ & 74,2 & 68,1 & 61,5 & & 50,3 & 54,3 & 57,3 \\
\hline Pueblos grandes & $2.000-19.999$ & 87,7 & 76,7 & 63,1 & & 50,9 & 60,1 & 61,1 \\
\hline Pueblos pequeños y población rural & $1-1.999$ & 99,5 & 96,2 & 71,6 & & 56,8 & 62,1 & $\mathbf{7 4 , 8}$ \\
\hline Total & & 74,0 & 67,6 & 56,5 & 43,7 & 48,2 & 53,7 & 51,4 \\
\hline
\end{tabular}


Fuente: Elaboración personal sobre la base del Censo 2010 (INDEC, 2012).

Nota $\left(^{*}\right)$ : Tasa de dependencia de población infantil (TDI) es el número de personas entre 0 y 13 por cada 100 personas mayores de 14 años.

Mientras que a nivel regional, los menores valores de la tasa de dependencia infantil se presentan en las regiones Pampeana y Patagonia, los valores más elevados pertenecen a las regiones del Norte.

El cruce de ambas dimensiones mencionadas anteriormente, nos permite determinar los valores extremos en este indicador. Los valores más bajos se dan en las ciudades grandes de las regiones Metropolitana y Pampeana (43,7 infantes por cada 100 personas potencialmente activas). Mientras que los valores más altos se ubican en los pueblos pequeños de las regiones Noreste y Noroeste (99,5 y 96,2 dependientes menores por cada cien personas en edad laboral, respectivamente).

Esta heterogeneidad presente en los valores del índice de dependencia demográfica de los menores, ejerce una diferencial en la carga económica a nivel espacial en el territorio nacional, por ejemplo en el gasto privado de los hogares en el rubro educativo.

De manera de aislar el efecto de la desigual estructura demográfica a nivel territorial sobre la oferta de empleo, definiremos la tasa de actividad "neta" que relaciona la PEA con la población mayor de 14 años y más.
Resulta evidente que el valor de la tasa neta será siempre superior a la bruta, ya que solo refleja la intensidad de la participación laboral de las personas que se encuentran en edad de trabajar. De ahora en adelante, cuando hagamos referencia a la tasa de actividad, estaremos considerando la tasa de actividad neta.

A continuación, analizaremos la relación entre la PEA y la población no económicamente activa (en adelante PNEA).Esta puede expresarse como la cantidad de trabajadores económicamente activos por cada trabajador inactivo.

A nivel nacional para el año 2010 ésta relación presenta un valor promedio de 1,76trabajadores potencialmente activos por cada trabajador inactivo.

Si consideramos el comportamiento en función de las categorías urbanas, el cociente PEA/PNEA presenta una relación inversa. El valor más alto, que es de 2,04 trabajadores económicamente activos por cada trabajador inactivo, se encuentra en las ciudades grandes y luego se observa como el valor de este indicador comienza a descender hasta alcanzar su mínimo de 1,29, en el caso de los pueblos pequeños. Ver Tabla3.

Tabla3: Relación PEA/NPEA, según categorías urbanas y regiones. Argentina 2010.

\begin{tabular}{|l|c|c|c|c|c|c|c|c|}
\hline \multirow{2}{*}{ Categorías urbanas } & \multirow{2}{*}{ Tamaño (hab) } & \multirow{6}{c|}{ Relación PEA/NPEA } \\
\cline { 5 - 9 } & & NEA & NOA & Cuyo & Metropolitana & Pampeana & Patagonia & \multirow{2}{*}{ Total } \\
\hline Metrópolis y Ciudades Grandes & $>1.000 .000$ & & & & 2,06 & 1,95 & & $\mathbf{2 , 0 4}$ \\
\hline ATIs grandes & $400.000-999.999$ & 1,53 & 1,59 & 1,68 & & 1,95 & 2,09 & $\mathbf{1 , 7 5}$ \\
\hline ATIS medias & $50.000-399.999$ & 1,41 & 1,63 & 1,68 & & 1,76 & 2,25 & $\mathbf{1 , 7 1}$ \\
\hline ATIS pequeñas & $20.000-49.999$ & 1,30 & 1,34 & 1,36 & & 1,68 & 1,99 & $\mathbf{1 , 5 7}$ \\
\hline Pueblos grandes & $2.000-19.999$ & 1,08 & 1,27 & 1,49 & & 1,64 & 2,07 & $\mathbf{1 , 4 9}$ \\
\hline Pueblos pequeños y población rural & $1-1.999$ & 0,98 & 0,96 & 1,46 & & 1,62 & 1,98 & $\mathbf{1 , 2 9}$ \\
\hline \multicolumn{2}{|c|}{ Total } & $\mathbf{1 , 2 5}$ & $\mathbf{1 , 3 8}$ & $\mathbf{1 , 6 0}$ & $\mathbf{2 , 0 6}$ & $\mathbf{1 , 7 7}$ & $\mathbf{2 , 1 0}$ & $\mathbf{1 , 7 6}$ \\
\hline
\end{tabular}

Fuente: Elaboración personal sobre la base del Censo 2010 (INDEC, 2012). 
A nivel regional, la relación entre la cantidad de trabajadores económicamente activos por cada inactivo, presenta una clara ventaja a favor de las regiones Pampeana, Metropolitana y Patagónica, por sobre las regiones del norte Argentino -mientras que en el caso de la región de Cuyo su situación es cercana al promedio nacional-.

Teniendo en cuenta la vinculación entre ambos factores, las mejores relaciones de dependencia se encuentran en las ATIS medias, ATIS grandes y pueblos grandes de la región Patagónica y en la Metrópolis del Gran Buenos Aires. En cambio los valores mínimos se ubican en las ATIS pequeñas, pueblos grandes y pueblos pequeños de las regiones Noreste y Noroeste.

Es importante destacar los cambios y la restructuración que se han dado en los espacios rurales argentinos en las últimas décadas. Específicamente desde los años noventa se produjo una reconfiguración territorial en las regiones extrapampeanas a partir de la apertura económica y del apoyo del estado a los grandes emprendimientos que se manifestaron en un fenómeno de modernización productiva y exclusión social (ROFMAN, 1999, p. 107136). El ingreso de las agroindustrias y el predominio de producciones altamente capitalizadas, acompañadas de tecnologías de capital intensivo introdujeron modificaciones en las características tradicionales de las economías regionales. De suma relevancia fue la irrupción de la expansión tecnológica-productiva de la especialización sojera, cuya consecuencia fue la generación de una enorme vulnerabilidad económica sobre los productores tradicionales (VALENZUELA, 2009). Este nuevo contexto en el espacio rural, vinculado al ritmo de los mercados globales y a la planificación de los negocios a escala mundial del capital internacional, ejerce una demanda de creciente competitividad, maximización de la rentabilidad, y por ende, minimización de los costos. Esto genera que la oferta laboral deba adaptarse a los nuevos requerimientos de producción flexible y de mayor precarización. La "modernización" de la agricultura genera cambios en el trabajo permanente, segmentación en el mercado de trabajo en cuanto a competencias, calificaciones y género - se establece un nuevo vínculo laboral, mediante la tercerización del factor trabajo y la utilización de empresas de servicios o contratistas de trabajos temporarios(CRAVIOTTI y PALACIOS, 2007) $-$

Lo rural no equivale ya a lo exclusivamente agrario, es decir, lo agrario no representa sólo a las producciones agrícolas y ganaderas, las vinculaciones entre la ciudad y el campo se intensifican cada vez más.

El espacio rural se revaloriza, entra en disputa por el uso del suelo, debido al avance de la mercantilización de nuevas demandas urbanas -se ofrece una nueva modalidad de residencia, que brinda esparcimiento y la tecnología necesaria a sectores urbanos de alto poder adquisitivo que les permite alejarse de los centros urbanos más concentrados con frecuencia, mediante emprendimientos inmobiliarios como, barrios privados, clubes de campo, estancias, etc. Este proceso fue potenciado por el capital invertido en especulación inmobiliaria y mediante el apoyo de los gobiernos locales deseosos de nuevas inversiones que ofrecen el reacomodamiento de la infraestructura necesaria- (TADEO, 2010).

\section{Diferencia entre la fuerza de trabajo masculina y femenina.}

Como mencionamos anteriormente, la tasa de actividad de una población resulta del cociente entre la población económicamente activa y la población en edad de trabajar. Para el año 2010, a nivel nacional, la tasa de actividad presentó un valor de 63,7\% -y considerando según sexos, fue de $76,1 \%$ en el caso de los hombres y $52,4 \%$ para las mujeres-.

En laTabla4 podemos apreciar la existencia de una relación directa entre el valor de la tasa de actividad -a nivel total y para cada uno de los sexosy las seis jerarquías urbanas. Es decir, en la categoría de mayor jerarquía urbana, que corresponde a las metrópolis y grandes ciudades, se registran las tasas de actividad más altas, mientras que en los pueblos 
pequeños se presentan los valores más bajos de participación laboral a nivel total.

Tabla4: Tasa de Actividad por sexo, según categorías urbanas y regiones. Año 2010. Población de 14 años y más (en \%).

\begin{tabular}{|c|c|c|c|c|}
\hline \multirow{2}{*}{ Categoria Urbana } & \multirow{2}{*}{ REGION } & \multicolumn{3}{|c|}{ Tasa de Actividad } \\
\hline & & Total & Masculina & Femenina \\
\hline \multicolumn{2}{|c|}{ Total } & 63,7 & 76,1 & 52,4 \\
\hline \multirow{3}{*}{$\begin{array}{c}\text { Metrópolis y Ciudades } \\
\text { Grandes }\end{array}$} & Sub total & 67,1 & 78,7 & 56,8 \\
\hline & Metropolitana & 67,4 & 79,0 & 56,9 \\
\hline & Pampeana & 66,1 & 77,1 & 56,2 \\
\hline \multirow{6}{*}{ ATIS grandes } & Sub total & 63,6 & 75,1 & 53,5 \\
\hline & Patagonia & 67,6 & 77,7 & 58,5 \\
\hline & Pampeana & 66,1 & 76,3 & 57,0 \\
\hline & Cuyo & 62,7 & 75,8 & 51,1 \\
\hline & $\mathrm{NOA}$ & 61,5 & 73,4 & 51,0 \\
\hline & NEA & 60,5 & 72,0 & 50,4 \\
\hline \multirow{6}{*}{ ATIS medias } & Sub total & 63,2 & 74,9 & 52,5 \\
\hline & Patagonia & 69,2 & 79,6 & 59,2 \\
\hline & Pampeana & 63,7 & 75,8 & 52,8 \\
\hline & Cuyo & 62,6 & 74,8 & 51,6 \\
\hline & $\mathrm{NOA}$ & 62,0 & 73,1 & 52,0 \\
\hline & NEA & 58,5 & 70,9 & 47,5 \\
\hline \multirow{6}{*}{ ATIS pequeñas } & Sub total & 61,1 & 74,3 & 49,1 \\
\hline & Patagonia & 66,5 & 77,7 & 55,8 \\
\hline & Pampeana & 62,6 & 75,5 & 51,1 \\
\hline & Cuyo & 57,7 & 72,7 & 44,2 \\
\hline & NOA & 57,3 & 70,3 & 45,2 \\
\hline & NEA & 56,5 & 71,7 & 42,7 \\
\hline \multirow{6}{*}{ Pueblos grandes } & Sub total & 59,9 & 73,5 & 47,2 \\
\hline & Patagonia & 67,4 & 78,8 & 56,3 \\
\hline & Pampeana & 62,1 & 75,4 & 49,8 \\
\hline & Cuyo & 59,8 & 74,9 & 45,5 \\
\hline & NOA & 55,9 & 69,6 & 43,0 \\
\hline & NEA & 52,0 & 67,3 & 37,7 \\
\hline \multirow{6}{*}{$\begin{array}{c}\text { Pueblos pequeños y } \\
\text { población rural }\end{array}$} & Sub total & 56,3 & 72,7 & 37,7 \\
\hline & Patagonia & 66,4 & 79,0 & 50,8 \\
\hline & Pampeana & 61,8 & 78,3 & 43,1 \\
\hline & Cuyo & 59,4 & 78,2 & 39,3 \\
\hline & NEA & 49,5 & 66,8 & 29,5 \\
\hline & NOA & 49,1 & 64,2 & 32,3 \\
\hline
\end{tabular}

Fuente: Elaboración personal sobre la base del Censo 2010 (INDEC, 2012).

También podemos apreciar cómo la tasa de actividad presenta sensibles diferencias interregionales. Se destacan a nivel total como para cada uno de los sexos, las altas tasas de las regiones Patagonia, Metropolitana y Pampeana.

Vinculando el nivel de jerarquía urbana y la división regional, la tasa de actividad total mínima se encuentra en los pueblos pequeños de la región Noroeste, con un valor de tan sólo 49,1\%, mientras que el valor más alto corresponde a las ATIS grandes de la región Pampeana, que alcanzan una tasa de actividad de 69,2\%.

Teniendo en cuenta el sexo, los valores de la tasa de actividad presentan una vinculación diferente en relación con las categorías urbanas en las distintas regiones.

Las brechas en la tasa de actividad entre trabajadoras femeninas, son superiores a la de los hombres, considerando las mismas regiones. También sucede lo mismo si observamos cada una de las regiones pero distintos niveles de jerarquía urbana -las diferencias resultan mayores conforme que aumenta la escala urbana-.

En el caso de los hombres, las jerarquías urbanas serían el factor explicativo de su mayor participación sólo en el caso de las regiones del Norte $\mathrm{y}$, en menor medida, en la región Patagónica. Cabe destacar la baja variabilidad en las tasas de participación masculina -entre todos los cruces entre categoría urbana y regiones-, el valor del C.V. es de tan sólo $5,4 \%$.

Mientras que la jerarquía urbana ejerce una influencia significativa en la participación femenina, potenciada a su vez por el diferencial regional. El resultado de esta situación son valores de tasa de actividad femenina con alta heterogeneidad a nivel espacial, el C.V. alcanza un valor de 15,7\% -casi tres veces superior al caso de los hombres-.

En síntesis, conforme aumenta el nivel de concentración urbana, más diversificadas tienden a ser las estructuras ocupacionales en cada una de las regiones -esto implica un aumento de la participación del sector terciario en la actividad económica-, por tanto, más elevado resulta el nivel de feminización de la fuerza de trabajo.

La participación de las mujeres en la PEA total del año 2010 es del 42,8\%. En la Tabla 5 podemos apreciar que la tasa de actividad femenina es siempre inferior a la masculina, en todas las combinaciones posibles entre categorías urbanas y regiones. Como expresamos anteriormente, su distribución presenta un sesgo hacia los cruces entre las categorías urbanas de mayor jerarquía y las regiones más diversificadas -en las cuales adquiere mayor participación el sector terciario y aumenta la demanda de empleo femenino-.

Si en cambio consideramos el porcentaje de fuerza de trabajo de cada sexo en relación a su 
propia PEA, se verifica que en las categorías urbanas más elevadas -ciudades grandes, ATIS grandes y medias-, el porcentaje de fuerza de trabajo femenina en relación a la PEA femenina supera el valor de esta relación en los hombres. Mientras que en las ATIS pequeñas, pueblos grandes, pueblos pequeños y población rural, esta relación no sólo se invierte sino que se incrementa la diferencia a favor de los hombres, conforme descendemos en el nivel de jerarquía urbana.

Tabla5- Participación de cada sexo en la fuerza de trabajo, según categorías urbanas y regiones. Año 2010. Población de 14 años y más (en \%).

\begin{tabular}{|c|c|c|c|c|c|c|c|}
\hline \multirow{2}{*}{ Categoría Urbana } & \multirow{2}{*}{ REGION } & \multicolumn{6}{|c|}{ Participación (en \%) } \\
\hline & & Varones & Mujeres & Total & Varones & Mujeres & Total \\
\hline \multicolumn{2}{|l|}{ Total } & 57,2 & 42,8 & 100,0 & 100,0 & 100,0 & 100,0 \\
\hline \multirow{3}{*}{ Metrópolis y Ciudades Grandes } & Subtotal & 55,4 & 44,6 & 100,0 & 42,5 & 45,6 & 43,8 \\
\hline & Metropolitana & 55,5 & 44,5 & 100,0 & 35,7 & 38,2 & 36,7 \\
\hline & Pampeana & 54,9 & 45,1 & 100,0 & 6,8 & 7,4 & 7,1 \\
\hline \multirow{6}{*}{ ATIS grandes } & Subtotal & 55,4 & 44,6 & 100,0 & 14,0 & 15,0 & 14,4 \\
\hline & Pampeana & 54,3 & 45,7 & 100,0 & 4,7 & 5,3 & 5,0 \\
\hline & Cuyo & 56,7 & 43,3 & 100,0 & 3,4 & 3,4 & 3,4 \\
\hline & NOA & 55,9 & 44,1 & 100,0 & 3,1 & 3,3 & 3,2 \\
\hline & NEA & 55,9 & 44,1 & 100,0 & 1,7 & 1,8 & 1,7 \\
\hline & Patagonia & 54,6 & 45,4 & 100,0 & 1,0 & 1,2 & 1,1 \\
\hline \multirow{6}{*}{ ATIS medias } & Subtotal & 56,4 & 43,6 & 100,0 & 14,9 & 15,4 & 15,1 \\
\hline & Pampeana & 56,3 & 43,7 & 100,0 & 7,0 & 7,2 & 7,1 \\
\hline & NOA & 56,0 & 44,0 & 100,0 & 3,0 & 3,1 & 3,1 \\
\hline & NEA & 57,0 & 43,0 & 100,0 & 1,9 & 1,9 & 1,9 \\
\hline & Patagonia & 56,3 & 43,7 & 100,0 & 1,8 & 1,9 & 1,8 \\
\hline & Cuyo & 56,8 & 43,2 & 100,0 & 1,2 & 1,2 & 1,2 \\
\hline \multirow{6}{*}{ ATIS pequeñas } & Subtotal & 57,9 & 42,1 & 100,0 & 8,0 & 7,7 & 7,9 \\
\hline & Pampeana & 57,1 & 42,9 & 100,0 & 4,2 & 4,3 & 4,2 \\
\hline & NEA & 60,4 & 39,6 & 100,0 & 1,2 & 1,1 & 1,2 \\
\hline & NOA & 59,0 & 41,0 & 100,0 & 1,1 & 1,0 & 1,1 \\
\hline & Patagonia & 57,0 & 43,0 & 100,0 & 1,0 & 1,1 & 1,0 \\
\hline & Cuyo & 59,6 & 40,4 & 100,0 & 0,4 & 0,3 & 0,4 \\
\hline \multirow{6}{*}{ Pueblos grandes } & Subtotal & 59,3 & 40,7 & 100,0 & 11,8 & 10,8 & 11,4 \\
\hline & Pampeana & 58,2 & 41,8 & 100,0 & 6,5 & 6,3 & 6,4 \\
\hline & NOA & 60,5 & 39,5 & 100,0 & 1,8 & 1,6 & 1,7 \\
\hline & NEA & 62,4 & 37,6 & 100,0 & 1,6 & 1,3 & 1,4 \\
\hline & Patagonia & 57,9 & 42,1 & 100,0 & 1,0 & 1,0 & 1,0 \\
\hline & Cuyo & 60,9 & 39,1 & 100,0 & 0,8 & 0,7 & 0,7 \\
\hline \multirow{6}{*}{ Pueblos pequeños y población rural } & Subtotal & 68,5 & 31,5 & 100,0 & 8,9 & 5,5 & 7,4 \\
\hline & Pampeana & 67,2 & 32,8 & 100,0 & 3,6 & 2,3 & 3,1 \\
\hline & NOA & 68,7 & 31,3 & 100,0 & 1,9 & 1,2 & 1,6 \\
\hline & NEA & 72,5 & 27,5 & 100,0 & 1,6 & 0,8 & 1,2 \\
\hline & Cuyo & 68,1 & 31,9 & 100,0 & 1,2 & 0,8 & 1,0 \\
\hline & Patagonia & 65,9 & 34,1 & 100,0 & 0,6 & 0,4 & 0,5 \\
\hline
\end{tabular}

Fuente: Elaboración personal sobre la base del Censo 2010 (INDEC, 2012). 
Cabe destacar el bajo porcentaje de trabajadoras femeninas sobre el total de su PEA, en los pueblos pequeños; tansólo 5,5\%, en comparación con los hombres en los que esta participación es de $8,9 \%$. A nivel regional el porcentaje de trabajadores femeninos supera al masculino en relación a la PEA total respectiva de cada sexo, sólo en el área Metropolitana.

Con respecto a la participación en el total de la fuerza de trabajo por parte de cada sexo, la máxima participación femenina (o la menor participación relativa masculina) se da en las ATIS grandes Pampeanas, mientras que la mínima participación relativa femenina ( o la mayor participación masculina sobre el total) se da en los pueblos pequeños del Noreste.

\section{Estructura educativa de la fuerza de trabajo}

En este punto nos abocamos al análisis del nivel deber tura educativo formal de la PEA total, masculina y femenina.

\begin{tabular}{|c|c|c|c|c|c|c|c|c|c|c|c|c|c|}
\hline \multirow[b]{3}{*}{ Categoria Urbana } & \multirow[b]{3}{*}{ Región } & \multicolumn{12}{|c|}{ Máximo Nivel de Instrucción Alcanzado de la PEA. En \% } \\
\hline & & \multicolumn{4}{|c|}{ Varones } & \multicolumn{4}{|c|}{ Mujeres } & \multicolumn{4}{|c|}{ Total } \\
\hline & & \begin{tabular}{|c|} 
Hasta \\
Secundario \\
incompleto
\end{tabular} & \begin{tabular}{|c|}
$\begin{array}{c}\text { Secundario } \\
\text { completo a } \\
\text { Superior } \\
\text { incompleto }\end{array}$ \\
\end{tabular} & \begin{tabular}{|l|}
$\begin{array}{l}\text { Superior } \\
\text { completo }\end{array}$ \\
\end{tabular} & Total & \begin{tabular}{|c|} 
Hasta \\
Secundario \\
incompleto
\end{tabular} & \begin{tabular}{|c|}
$\begin{array}{c}\text { Secundario } \\
\text { completo a } \\
\text { Superior } \\
\text { incompleto }\end{array}$ \\
\end{tabular} & $\begin{array}{l}\text { Superior } \\
\text { completo }\end{array}$ & Total & $\begin{array}{c}\text { Hasta } \\
\text { Secundario } \\
\text { incompleto }\end{array}$ & \begin{tabular}{|c|} 
Secundario \\
completo a \\
Superior \\
incompleto
\end{tabular} & $\begin{array}{l}\text { Superior } \\
\text { completo }\end{array}$ & Total \\
\hline \multicolumn{2}{|l|}{ TOTAL } & 69,8 & 22,8 & 7,4 & 100,0 & 64,8 & 24,5 & 10,7 & 100,0 & 67,3 & 23,7 & 9,1 & 100,0 \\
\hline \multirow{3}{*}{$\begin{array}{c}\text { Metrópolis y Ciudades } \\
\text { Grandes }\end{array}$} & TOTAL & 65,0 & 26,2 & 8,8 & 100,0 & 60,2 & 27,6 & 12,2 & 100,0 & 62,5 & 26,9 & 10,6 & 100,0 \\
\hline & Metropolitana & 65,5 & 25,8 & 8,6 & 100,0 & 60,7 & 27,3 & 11,9 & 100,0 & 63,0 & 26,6 & 10,3 & 100,0 \\
\hline & Pampeana & 62,1 & 28,0 & 9,8 & 100,0 & 57,3 & 28,9 & 13,8 & 100,0 & 59,6 & 28,5 & 11,9 & 100,0 \\
\hline \multirow{6}{*}{ ATIS grandes } & TOTAL & 64,7 & 26,8 & 8,5 & 100,0 & 59,3 & 28,4 & 12,3 & 100,0 & 61,9 & 27,6 & 10,5 & 100,0 \\
\hline & NEA & 67,4 & 25,8 & 6,9 & 100,0 & 61,9 & 27,4 & 10,8 & 100,0 & 64,5 & 26,6 & 8,9 & 100,0 \\
\hline & NOA & 65,8 & 26,7 & 7,6 & 100,0 & 60,4 & 28,3 & 11,3 & 100,0 & 62,9 & 27,5 & 9,5 & 100,0 \\
\hline & Cuyo & 66,2 & 25,4 & 8,4 & 100,0 & 61,1 & 27,0 & 11,9 & 100,0 & 63,5 & 26,2 & 10,3 & 100,0 \\
\hline & Pampeana & 61,1 & 29,0 & 10,0 & 100,0 & 56,0 & 29,9 & 14,1 & 100,0 & 58,4 & 29,5 & 12,1 & 100,0 \\
\hline & Patagonia & 67,9 & 24,3 & 7,7 & 100,0 & 60,1 & 28,2 & 11,7 & 100,0 & 63,9 & 26,3 & 9,8 & 100,0 \\
\hline \multirow{6}{*}{ ATIS medias } & TOTAL & 69,3 & 23,6 & 7,1 & 100,0 & 64,1 & 24,9 & 11,0 & 100,0 & 66,6 & 24,3 & 9,1 & 100,0 \\
\hline & NEA & 71,9 & 21,9 & 6,2 & 100,0 & 67,5 & 23,4 & 9,1 & 100,0 & 69,6 & 22,7 & 7,7 & 100,0 \\
\hline & NOA & 69,8 & 24,0 & 6,2 & 100,0 & 63,3 & 26,6 & 10,0 & 100,0 & 66,4 & 25,4 & 8,2 & 100,0 \\
\hline & Cuyo & 69,3 & 23,9 & 6,9 & 100,0 & 64,0 & 25,3 & 10,6 & 100,0 & 66,5 & 24,6 & 8,8 & 100,0 \\
\hline & Pampeana & 67,2 & 24,9 & 7,9 & 100,0 & 62,2 & 25,5 & 12,3 & 100,0 & 64,6 & 25,2 & 10,2 & 100,0 \\
\hline & Patagonia & 70,1 & 22,9 & 7,0 & 100,0 & 65,0 & 24,0 & 11,0 & 100,0 & 67,5 & 23,5 & 9,1 & 100,0 \\
\hline \multirow{6}{*}{ ATIS pequeñas } & TOTAL & 74,5 & 19,9 & 5,6 & 100,0 & 68,5 & 21,3 & 10,2 & 100,0 & 71,4 & 20,6 & 8,0 & 100,0 \\
\hline & NEA & 79,7 & 15,7 & 4,6 & 100,0 & 75,2 & 16,7 & 8,2 & 100,0 & 77,4 & 16,2 & 6,4 & 100,0 \\
\hline & NOA & 76,3 & 19,4 & 4,3 & 100,0 & 69,6 & 21,5 & 8,8 & 100,0 & 72,9 & 20,5 & 6,6 & 100,0 \\
\hline & \begin{tabular}{|l|} 
Cuyo \\
\end{tabular} & 76,5 & 18,2 & 5,3 & 100,0 & 70,2 & 19,8 & 10,1 & 100,0 & 73,2 & 19,0 & 7,8 & 100,0 \\
\hline & Pampeana & 72,1 & 21,5 & 6,4 & 100,0 & 66,1 & 22,5 & 11,4 & 100,0 & 69,0 & 22,0 & 9,0 & 100,0 \\
\hline & Patagonia & 74,9 & 19,6 & 5,5 & 100,0 & 68,1 & 22,7 & 9,2 & 100,0 & 71,4 & 21,1 & 7,4 & 100,0 \\
\hline \multirow{6}{*}{ Pueblos grandes } & TOTAL & 78,0 & 17,2 & 4,8 & 100,0 & 71,7 & 19,1 & 9,2 & 100,0 & 74,8 & 18,2 & 7,1 & 100,0 \\
\hline & NEA & 83,3 & 12,7 & 4,0 & 100,0 & 79,2 & 13,7 & 7,1 & 100,0 & 81,2 & 13,2 & 5,6 & 100,0 \\
\hline & NOA & 80,0 & 16,2 & 3,9 & 100,0 & 73,7 & 18,9 & 7,5 & 100,0 & 76,8 & 17,5 & 5,7 & 100,0 \\
\hline & Cuyo & 80,2 & 15,9 & 3,9 & 100,0 & 74,1 & 18,6 & 7,3 & 100,0 & 77,1 & 17,2 & 5,7 & 100,0 \\
\hline & Pampeana & 75,8 & 18,9 & 5,3 & 100,0 & 69,1 & 20,4 & 10,5 & 100,0 & 72,4 & 19,7 & 8,0 & 100,0 \\
\hline & Patagonia & 76,2 & 17,9 & 5,9 & 100,0 & 69,4 & 21,0 & 9,5 & 100,0 & 72,8 & 19,5 & 7,7 & 100,0 \\
\hline \multirow{6}{*}{$\begin{array}{c}\text { Pueblos pequeños y } \\
\text { población rural }\end{array}$} & TOTAL & 88,1 & 9,7 & 2,3 & 100,0 & 83,9 & 11,8 & 4,3 & 100,0 & 86,1 & 10,7 & 3,2 & 100,0 \\
\hline & NEA & 93,3 & 5,5 & 1,3 & 100,0 & 91,7 & 6,2 & 2,1 & 100,0 & 92,5 & 5,8 & 1,6 & 100,0 \\
\hline & NOA & 91,0 & 7,5 & 1,5 & 100,0 & 87,6 & 9,6 & 2,9 & 100,0 & 89,4 & 8,5 & 2,1 & 100,0 \\
\hline & Cuyo & 88,1 & 9,8 & 2,1 & 100,0 & 83,6 & 12,4 & 4,0 & 100,0 & 85,9 & 11,1 & 3,0 & 100,0 \\
\hline & Pampeana & 83,9 & 12,9 & 3,1 & 100,0 & 78,1 & 15,6 & 6,3 & 100,0 & 81,2 & 14,2 & 4,7 & 100,0 \\
\hline & Patagonia & 83,9 & 12,3 & 3,9 & 100,0 & 78,5 & 15,0 & 6,6 & 100,0 & 81,4 & 13,5 & 5,1 & 100,0 \\
\hline
\end{tabular}

Operacionalizaremos a partir de comparar el grado educativo máximo alcanzando por las fuerzas laborales, y el efecto que genera en términos de desigualdad, el nivel de jerarquía urbanas en cada ámbito regional.

La Argentina se ha destacado entre los países latinoamericanos por su temprano y efectivo proceso de universalización de la enseñanza de nivel primario, que se consolida sobre todo a partir de mitad del siglo pasado.

En la Tabla 6 se observa, tanto a nivel total como para hombres y mujeres, una relación directa las categorías de jerarquías urbanas, así como un diferencial en favor de las regiones Metropolitana, Pampeana y Patagónica, sobre las restantes regiones del país.

Tabla 6: Máximo Nivel de Instrucción Alcanzado de la PEA de cada sexo, según categorías urbanas y regiones. Año 2010. En porcentaje. entre mayor nivel educativo alcanzado por la PEA y 
Fuente: Elaboración personal sobre la base del Censo 2010 (INDEC, 2012).

Considerando las fuerzas laborales totales respecto a las categorías de jerarquías urbanas, en las ATIS grandes se encuentra el porcentaje más elevado de PEA con nivel secundario completo o más, con un valor de $38,1 \%$, mientras que en los pueblos pequeños se encuentra el valor mínimo, de tan sólo $13,9 \%$. En el caso de los hombres y mujeres también ocurre que los valores máximos y mínimos se ubican en las mismas categorías urbanas. Presentando las brechas máximas un valor de 24,2 p.p. para el total; 23,4 p.p. en los hombres y 24,6 p.p. para las mujeres.

Al interior de cada una de las regiones se comprueba que conforme nos desplazamos hacia mayores niveles de jerarquía urbana, las brechas disminuyen.

El porcentaje más elevado de la PEA -tanto a nivel total, como para cada uno de los sexos-, que terminaron el nivel secundario o más, se encuentra en la región Metropolitana $(37,0 ; 34,5 \%$ y 39,3\%, respectivamente). Mientras que el menor porcentaje se registra en la región Nordeste, con un valor de $23,5 \%$ para el nivel total, $21,1 \%$ en los hombres y $25,9 \%$ en las mujeres.

\section{Conclusiones}

En este trabajo procedimos a analizar los cambios que presenta la oferta laboral, considerando diferentes niveles de jerarquía urbana en cada una de las regiones de Argentina.

El marco general en términos de distribución poblacional, es de una fuerte concentración en los aglomerados de mayor jerarquía urbana, así como también a nivel regional. El 71,3\% de la población de 14 años y más, y el $73,3 \%$ de la PEA reside en aglomerados con una población superior a los 50 mil habitantes, es decir se corresponde con los tres niveles superiores de jerarquía urbana: ciudades grandes; ATIS grandes y ATIS medias. Mientras que a nivel regional, el $67,6 \%$ de las personas con 14 años y más, y el 69,6\% de la PEA, se concentra en sólo dos regiones -Metropolitana y Pampeana-.

Los puntos más importantes han surgido de la comparación de la oferta de empleo mediante el entrecruzamiento del ranking de categorías urbanas y las distintas regiones, siendo los aspectos salientes:

- Relación inversa entre el nivel de escala urbana y el valor de relación de dependencia demográfica de los menores-en las ciudades más grandes las tasas de dependencia demográfica de los menores son las más bajas y a medida que se reduce el nivel de jerarquía urbana este indicador acrecienta su valor-

- Relación directa entreel nivel de jerarquía urbana y la relación PEA/NPEA -en las ciudades grandes se presentan los valores máximos, mientras que conforme nos desplazamos a una menor escala urbana este cociente disminuye hasta alcanzar su valor mínimo en los pueblos pequeños-.

- Relación directa entre el nivel jerarquía urbana y valor de la tasa de actividad total (a medida que se eleva el nivel de jerarquía urbana mayores resultan los valores de la tasa de participación laboral total, destacándose sensibles diferencias entre las regiones del país, es decir, a igual nivel de jerarquía urbana, las regiones de la Patagonia, Metropolitana y Pampeana presentan tasas más altas de participación poblacional en la actividad económica, que las restantes regiones).

- Relación directa entre el nivel educativo máximo alcanzado por la PEA y la jerarquía urbana tanto a nivel total, como para hombres y mujeres-

- Relación directa entre tasa de actividad laboral femenina y nivel de jerarquía urbana: cuanto más alto sea el nivel de jerarquía urbana en cada región, más elevada resulta la tasa de actividad laboral femenina, además este efecto se potencia en base a la diversidad de la estructura ocupacional de cada región -la mayor participación relativa del sector terciario en total productivo, es un factor determinante del aumento de la oferta de empleo femenina -. Lo que confirma la validez de nuestra hipótesis. 
La tendencia hacia una mayor diversificación de las actividades existentes, genera una disminución del peso relativo de la participación de los sectores primarios y secundarios, debido al incremento de los nuevos servicios que se generan en los mercados de mayor densidad económica. Este avance de un perfil productivo cada vez más relacionado a los servicios, presenta un mercado laboral en donde la oferta de empleo femenino es cada vez más importante.

Las regiones del norte -Noroeste y Noreste-, se destacan por presentar la mayor participación de la actividad primaria en la actividad económica total, lo que se vincula con la mayor participación del trabajo masculino, así como también estas condiciones expresan los valores más altos en la relación de dependencia demográfica infantil, las menores tasas de actividad total y los menores niveles educativos. Mientras que en iguales niveles de jerarquía urbana, en las regiones Patagonia y Pampeana, que presentan una demanda de empleo con una participación relevante del sector terciario, presentan una situación superadora en todos los indicadores demográficos mencionados.

Todo esto contribuye a explicar los diferenciales hallados en trabajos anteriores en donde se exploraba la relación entre calidad de vida y escala urbana por regiones (VELÁZQUEZ et al, 2014). En las ATIS medias y grandes de las regiones pampeana y patagónica se registran los mejores índices de calidad de vida -por combinación de indicadores socioeconómicos y ambientales-. Evidentemente la esfera laboral efectúa una contribución importante para explicar estas diferencias.

\section{Referências}

ARGENTINA. INDEC. Censo Nacional de Población, Familias y Viviendas 1970. Buenos Aires, 1974.

\author{
- Censo Nacional de Población y Vivienda \\ 1980, Buenos Aires, 1981.
}

Censo Nacional de Población y Vivienda 1991, Serie B. Buenos Aires, 1992.

Censo Nacional de Población, Hogares y Viviendas del año 2001, Buenos Aires, 2004.
Censo Nacional de Población, Hogares y Viviendas 2010. Censo del Bicentenario. Resultados definitivos. Serie B tomo 1 y 2, Buenos Aires, 2012.

CACOPARDO, C. y M. J. L. Cuando los hombres estaban ausentes: la familia del Interior de la Argentina decimonónica. En OTERO, Héctor y VELÁZQUEZ, Guillermo (comps.), Poblaciones argentinas. Estudios de demografía diferencial. Tandil: PROPIEP (CIG-IEHS), Universidad Nacional del Centro, 1997.

CRAVIOTTI, C. y PALACIOS, P. La trama detrás de la escena: los contratistas de servicios de mano de obra en la producción de frutas frescas de alto valor. En: Revista Estudios del Trabajo, 33, 2007, p. 3-32.

DEIE. Dirección de Estadística e Investigaciones Económicas. Disponible en: $<$ http://www.deie.mendoza.gov.ar/tematicas/censos/c ensos_digitalizados/Censos\%20Digitalizados/index.ht $\mathrm{ml}$. Acceso: Abril de 2016.

OTERO, H. Legalidad jurídica y legalidad estadística en el paradigma censal argentino, 1869-1914. En FRADKIN, R.; CAÑEDO, M. y MATEO, J. (comp.) Población y relaciones sociales en la campaña de Buenos Aires. Mar del Plata: GIHRR-UNMdP, 1999.

PALOMINO, H. Cambios ocupacionales y sociales en Argentina.1947-1985. Buenos Aires: CISEA, 1988.

RECCHINI DE LATTES, Z. y LATTES, A. La población de argentina. Buenos Aires: CICRED, 1975.

ROFMAN, A. Economías regionales. Modernización productiva y exclusión. En:Revista Realidad Económica, 162, 1999, p. 107-136.

TADEO, N. Los espacios rurales en la argentina actual. Nuevos enfoques y perspectivas de análisis desde la geografía rural. En: Mundo agrario, 10, 20, 2010.

VALENZUELA, C. Territorio, identidad y permanencia en nuevos contextos productivos. La 'resistencia' de la agricultura familiar tradicional en el Chaco, Argentina. En: Economía, Sociedad yTerritorio, 30, 2009, p. 397-433.

VAPÑARSKY, C. y GOROJOVSKY, N. El crecimiento urbano en la Argentina. Buenos Aires: Grupo Editor Latinoamericano-IIED, 1990.

VELÁZQUEZ, G. Geografía y Bienestar. Buenos Aires: Eudeba, 2008.

VELÁZQUEZ, G;MIKKELSEN, C; LINARES, S. y CELEMÍN, J. P. Geografía y calidad de vida en Argentina. Ranking del bienestar por departamentos (2010). Tandil, IGEHCS/CIG, 2014. 